\title{
Microstructured Switchable Mirror for Polar Molecules
}

\author{
Stephan A. Schulz, Hendrick L. Bethlem, Jacqueline van Veldhoven, Jochen Küpper, Horst Conrad, and Gerard Meijer \\ Fritz-Haber-Institut der Max-Planck-Gesellschaft, Faradayweg 4-6, D-14195 Berlin, Germany \\ and FOM-Institute for Plasmaphysics Rijnhuizen, P.O. Box 1207, NL-3430 BE Nieuwegein, The Netherlands
}

(Received 27 January 2004; published 9 July 2004)

\begin{abstract}
By miniaturizing electrode geometries high electric fields can be produced using modest voltages. A planar array of $20 \mu \mathrm{m}$ wide gold electrodes, spaced $20 \mu \mathrm{m}$ apart, is made on a sapphire substrate. A voltage difference of up to $350 \mathrm{~V}$ is applied to adjacent electrodes, generating an electric field that decreases exponentially with distance from the substrate. This microstructured array can be used as a mirror for polar molecules and can be rapidly switched on and off. This is demonstrated by retroreflecting a beam of state-selected ammonia molecules with a forward velocity of about $30 \mathrm{~m} / \mathrm{s}$.
\end{abstract}

DOI: 10.1103/PhysRevLett.93.020406

Miniaturizing current carrying structures has proven to be a very successful strategy for atom optics [1,2]. Microfabricated wires on surfaces allow one to exert extremely high magnetic forces on atoms using only moderate currents. A variety of microfabricated atom optical elements such as mirrors [3], guides [4], conveyer belts, and traps [5] have been realized. The integration of many of these devices into one circuit offers novel and exciting possibilities for quantum computation and atom interferometry [6,7].

Miniaturizing charge carrying structures to manipulate polar molecules is equally promising. Using microstructured electrodes large electric fields and large field gradients can be generated with only moderate voltages. The interaction of polar molecules with electric fields is orders of magnitude stronger than the interaction of atoms with magnetic fields, and one can easily construct potentials on the order of a Kelvin. This allows one to design microstructured electrodes to manipulate cold polar molecules as produced, for instance, via buffer gas loading [8], Stark deceleration [9], collisions [10], or photoassociation [11]. The rotational and vibrational degrees of freedom as well as the (anisotropic) dipole-dipole interaction of polar molecules offer novel possibilities for interferometry and quantum computation [12].

In this Letter, we experimentally demonstrate a microstructured switchable mirror for polar molecules. It is well known that a planar array of equidistant electrodes with a voltage difference between adjacent electrodes produces an electric field that decays exponentially with the distance from the surface. Such an array can therefore be used as an electrostatic mirror for polar molecules in so-called low-field seeking quantum states [13]. The principle of an electrostatic mirror was first discussed by Gordon as a means to select slow molecules [14]. Later this geometry was discussed in much more detail by Opat and co-workers $[15,16]$, who experimentally demonstrated an electrostatic mirror by reflecting a beam of chloromethane $\left(\mathrm{CH}_{3} \mathrm{Cl}\right)$ from it at grazing angles of incidence. In the experiments reported here we demonstrate reflection of a cold beam of state-selected polar molecules
PACS numbers: 03.75.Be, 33.55.Be, 33.80.Ps, 39.10.+j

from a microstructured electrostatic mirror under normal incidence. The mirror consists of an interdigitated structure of $20 \mu \mathrm{m}$ wide gold electrodes, $20 \mu \mathrm{m}$ apart, on a planar sapphire substrate, that can withstand a voltage difference of up to $350 \mathrm{~V}$ between adjacent electrodes.

In Fig. 1 a scheme of the experimental setup is shown. A detailed description of the molecular beam machine, and particularly of the deceleration of a beam of ammonia molecules, is given elsewhere [17]. In the experiments reported here, a pulsed beam of deuterated ammonia molecules is decelerated from 280 to $29 \mathrm{~m} / \mathrm{s}$ using a

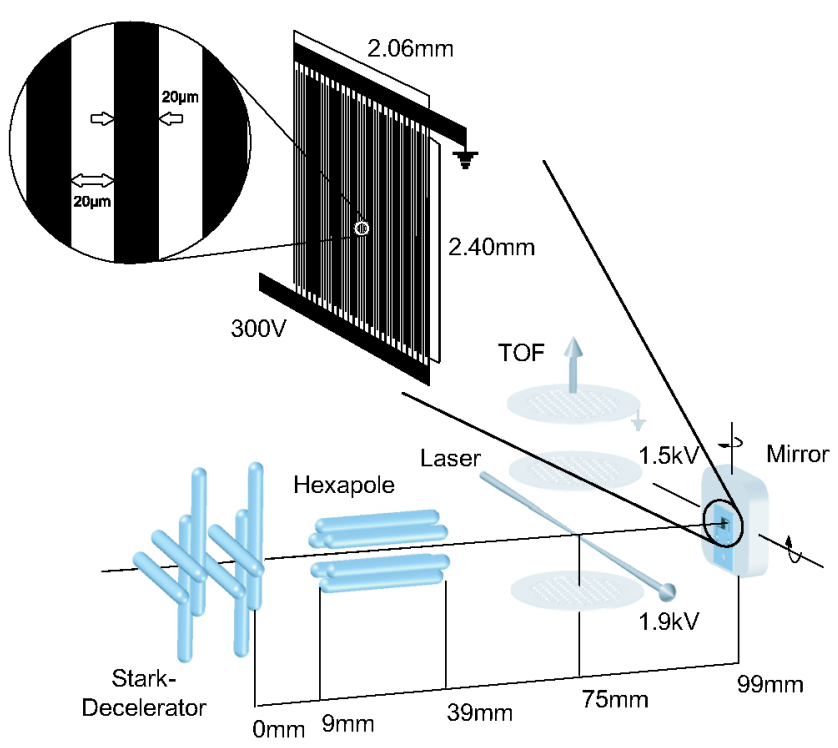

FIG. 1 (color online). Scheme of the experimental setup. A pulsed beam of ammonia molecules is state selected and decelerated using a $52 \mathrm{~cm}$ long Stark decelerator, of which only the last four stages are shown. The slow beam exiting the decelerator is collimated by a hexapole lens. About $75 \mathrm{~mm}$ behind the decelerator the ammonia molecules can be detected via resonant ionization with a pulsed UV laser. The ion intensity is mass-selectively recorded using a linear time-of-flight (TOF) setup. The microstructured mirror, shown enlarged (see top of figure), is positioned $24 \mathrm{~mm}$ further downstream. The retroreflected molecules pass through the detection zone once more. 
$52 \mathrm{~cm}$ long Stark decelerator consisting out of 94 equidistant electric field stages; in Fig. 1 only the last four stages of the Stark decelerator are shown. Only ammonia molecules in the upper component of the $|J, K\rangle=|1,1\rangle$ inversion doublet are decelerated [17]. At the exit of the decelerator, the package of slow ammonia molecules has a spatial extent of about $1.5 \mathrm{~mm}$ along the molecular beam axis and is about $2 \times 2 \mathrm{~mm}^{2}$ in the transverse direction. The full width at half maximum (FWHM) of the velocity spread in the forward direction is about $8 \mathrm{~m} / \mathrm{s}$, corresponding to a longitudinal temperature of $28 \mathrm{mK}$. In the transverse direction the FWHM velocity spread is slightly less, about $5 \mathrm{~m} / \mathrm{s}$, corresponding to a transverse temperature of $11 \mathrm{mK}$. After exiting the decelerator the slow beam is collimated using a pulsed electrostatic hexapole lens. The focal length is set such that the focal plane lies slightly behind the surface of the electrostatic mirror, thereby optimizing the signal of the retroreflected beam in the detection zone. About $75 \mathrm{~mm}$ behind the decelerator, the ammonia molecules pass through the detection zone. Using a pulsed tunable UV laser, ${ }^{14} \mathrm{ND}_{3}$ molecules in the upper component of the $|J, K\rangle=|1,1\rangle$ inversion doublet are selectively ionized in a $(2+1)$-resonance enhanced multiphoton ionization (REMPI) process. The ions are subsequently mass-selectively detected in a compact linear time-of-flight (TOF) setup. The intensity of the parent ion signal, which is directly proportional to the density of ammonia molecules in this particular quantum state in the detection zone, is recorded. The retroreflected molecules pass through the same detection zone once more. The microstructure, mounted on a manipulator to be able to optimize its position and angles, is placed $24 \mathrm{~mm}$ further downstream. The microstructured array of electrodes is made by depositing a $0.3 \mu \mathrm{m}$ thick gold layer onto a planar sapphire substrate with a Pt/Ti-type contact layer. After a lithographic process, $20 \mu \mathrm{m}$ wide lines are removed in an etching procedure to obtain the desired electrode geometry. The total area of the structure is $2.40 \times 2.06 \mathrm{~mm}^{2}$, only large enough to reflect the central portion of the impinging molecular beam. The microstructured array is shown enlarged (see top of Fig. 1). In the experiment a voltage difference of up to $350 \mathrm{~V}$ is applied between adjacent electrodes, corresponding to an electric field of over $175 \mathrm{kV} / \mathrm{cm}$ at the substrate. These electric fields are reproducibly obtained after a conditioning process of several hours. Voltage differences exceeding $400 \mathrm{~V}$ resulted in severe damage of the structure. The electric field strength that can be achieved on the microstructure is surprisingly large. This has also been noted by Schmiedmayer and co-workers in recent experiments in which they manipulated cold atoms with combined electric and magnetic fields [18]. That such high electric fields can be achieved is most likely due to the relatively small absolute voltages that are required; when a discharge occurs, the electrons only get a limited kinetic energy, insufficient to damage the structure or to initiate ava- lanche processes. It is anticipated that even higher electric fields can be obtained using smaller structures.

In the upper part of Fig. 2 a plot of the magnitude of the electric field $E$ in front of the surface of the microstructured electrodes is shown. This field is calculated using a finite element program and in the calculation a voltage difference between the electrodes of $300 \mathrm{~V}$ is assumed. Close to the substrate the electric field is highly corrugated but at distances from the surface corresponding to a fraction of the periodicity of the electrode geometry the magnitude of the electric field is nearly independent of position relative to the electrodes. In this region the electric field appears almost flat, and decays exponentially with distance from the electrodes. The electric field of the actual electrode geometry can be compared to the analytical expression for the electric field resulting from an array of equally spaced line electrodes [16]. For distances $z$ far away $(z \gg a / 4 \pi)$ from the plane of the line electrodes (taken to be at $z=0$ ) the electric field decays exponentially and can be approximated by [16]:

$$
E(z)=\frac{4 \pi V}{a} \exp ^{-2 \pi z / a},
$$

where $V$ is the voltage difference between adjacent
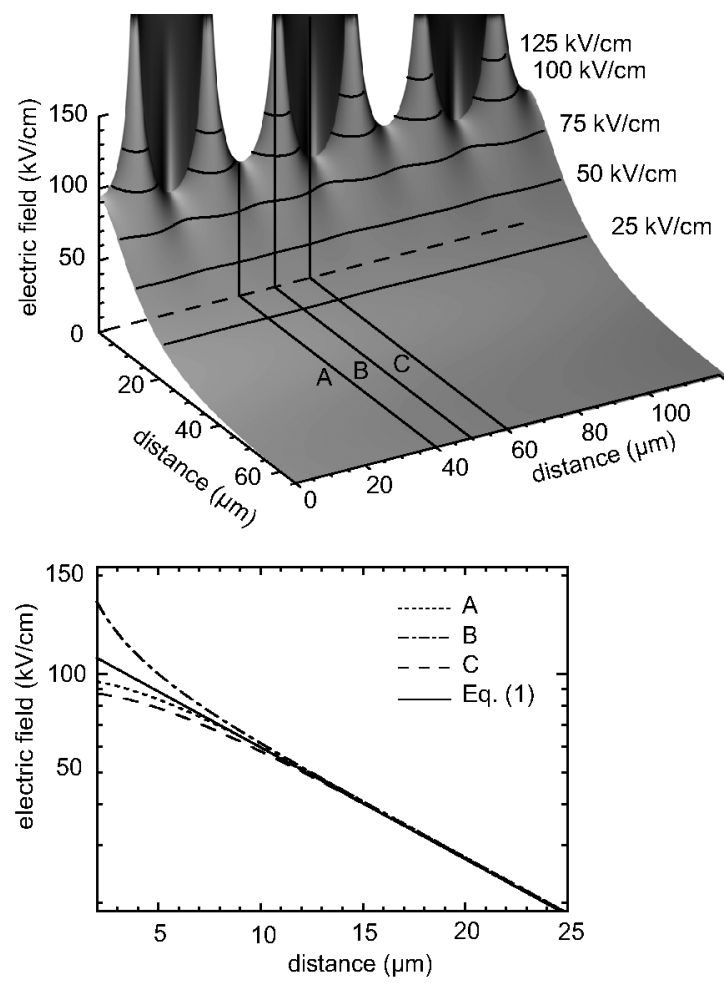

FIG. 2. Plot of the magnitude of the electric field in the plane perpendicular to the electrodes calculated using a finite element program. The voltage difference between adjacent electrodes is $300 \mathrm{~V}$. The electric field as a function of the distance from the surface along the lines $A, B$, and $C$ is shown on a logarithmic scale in the lower graph. The straight solid line indicated in this graph follows from the analytical expression, with $z$ replaced by $z+9.5 \mu \mathrm{m}$. 
electrodes and $a$ is the electrode period ( $80 \mu \mathrm{m}$ in our case). In the lower part of Fig. 2 the magnitude of the electric field along three different lines $A, B$, and $C$ (see upper part of Fig. 2) is shown on a logarithmic scale. For comparison, the electric field for an array of line electrodes, as given by the analytical approximation, is shown as well. Excellent agreement is obtained in the far field when the line electrodes are assumed to be positioned $9.5 \mu \mathrm{m}$ behind the actual electrode array, i.e., when $E(z+9.5 \mu \mathrm{m})$ is plotted.

Molecules in quantum states with a positive Stark effect, i.e., molecules in low-field seeking states, will gain potential energy while moving towards the plane of the microstructured electrodes. Molecules having insufficient kinetic energy to overcome the potential barrier will be reflected. Deuterated ammonia molecules in lowfield seeking (hyperfine) levels of the $|J, K\rangle=|1,1\rangle$ state with an initial velocity of $32 \mathrm{~m} / \mathrm{s}\left(E_{\mathrm{kin}} / k=1.2 \mathrm{~K}\right)$ will be turned around at an electric field strength of about $75 \mathrm{kV} / \mathrm{cm}$; molecules with an initial velocity of $26 \mathrm{~m} / \mathrm{s}$ $\left(E_{\mathrm{kin}} / k=0.8 \mathrm{~K}\right)$ will be reflected by an electric field of about $50 \mathrm{kV} / \mathrm{cm}$. The potential hill that the molecules experience is rather steep, and the difference in penetration depth between the fastest and the slowest molecules in the decelerated package of ammonia molecules is less than $10 \mu \mathrm{m}$. As this is very small compared to the total flight distance of the molecules, the mirror can be considered hard.

In Fig. 3 the arrival time distributions are shown for ammonia molecules recorded in the detection zone, $75 \mathrm{~mm}$ behind the decelerator, with (black curve) and without (gray curve) a voltage of $300 \mathrm{~V}$ applied to the microstructured electrodes. The package of decelerated

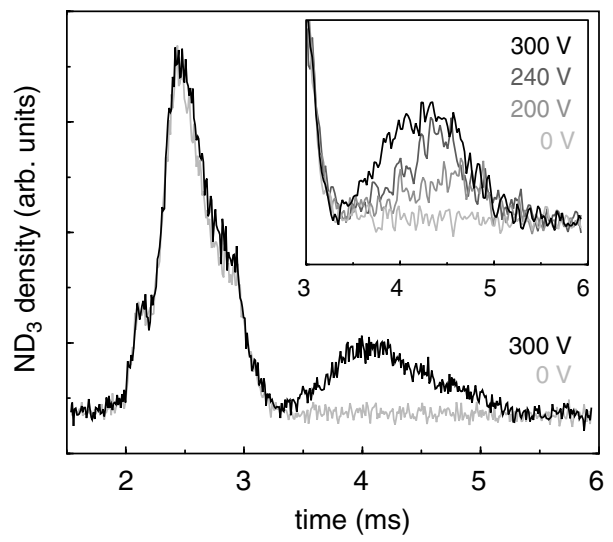

FIG. 3. Time-of-flight distribution of the ammonia molecules in the detection zone. The package of slow molecules passes through the detection zone about $2.5 \mathrm{~ms}$ after exiting the decelerator (the latter is taken as the origin of the time axis). Two sets of independent measurements are shown, with (black curve) and without (gray curve) voltage applied to the microstructured array; the retroreflected molecules travel an extra $48 \mathrm{~mm}$ before being detected at about $4 \mathrm{~ms}$. In the inset the arrival time distribution of the reflected molecules is shown for different voltages. molecules is seen to pass through the detection zone around $2.5 \mathrm{~ms}$ after exiting the decelerator. The reflected molecules travel an extra $48 \mathrm{~mm}$ before passing through the detection zone once more, about $4 \mathrm{~ms}$ after exiting the decelerator. As the initial spatial spread of the package of molecules is small compared to the total flight distance, the observed arrival time distribution directly mimics the longitudinal velocity distribution of the beam. From this, it is deduced that the beam indeed has an average velocity of $29 \mathrm{~m} / \mathrm{s}$ and a FWHM velocity spread of $8 \mathrm{~m} / \mathrm{s}$. Because of coupling of the transverse velocity with the longitudinal velocity in the decelerator, the divergence of the decelerated beam depends on the forward velocity [17]. This results in a non-Gaussian shape of the arrival time distribution, depending, among other things, on the focal length of the hexapole and on the total flight distance. Since the reflected molecules have a substantially longer flight path, there is a more stringent selection on the transverse velocity component for these molecules, and only retroreflected molecules in a relatively narrow translational distribution interval are detected. This results in an arrival time distribution of the reflected beam which is actually more Gaussian-like than that of the incoming beam. The observed signal intensity of the retroreflected beam is as expected for a reflectivity of unity, given the rather compact overall size of the mirror in combination with the more stringent requirements on the divergence of the reflected beam relative to the one of the incoming beam.

In the inset of Fig. 3 arrival time distributions for the retroreflected beam are shown with voltage differences of $0,200,240$, and $300 \mathrm{~V}$ applied to the microstructured electrodes. The distribution observed with a voltage of $300 \mathrm{~V}$ is identical to the one observed with $350 \mathrm{~V}$ (not shown). On lowering the voltage, the signal observed at early times (which results from the fastest molecules in the package) decreases. When the voltage is lowered to below $150 \mathrm{~V}$ hardly any retroreflected molecules are detected. This is expected, as faster molecules are able to climb higher up the potential hill, and some of them might even hit the substrate. In the latter case they might stick to the surface or scatter inelastically. Molecules that do not actually hit the surface but nevertheless approach the surface within several $\mu \mathrm{m}$ will be reflected over relatively large angles due to the corrugation of the potential hill. All these effects will result in a decrease in the number of state-selected reflected molecules in the detection zone. Note that so far we have described the interaction of the molecules with the electrostatic field purely classically, as balls rolling up and down a slightly corrugated hill. Given the rather large transverse velocity spread of the package of molecules that is reflected, and given the tens of $\mu \mathrm{m}$ sized electrodes that we have used, this approach is valid in the present study. However, when nanometer scaled electrodes are used in combination with (transversely) still colder packages of molecules the wave properties of the molecules will have to be taken into 


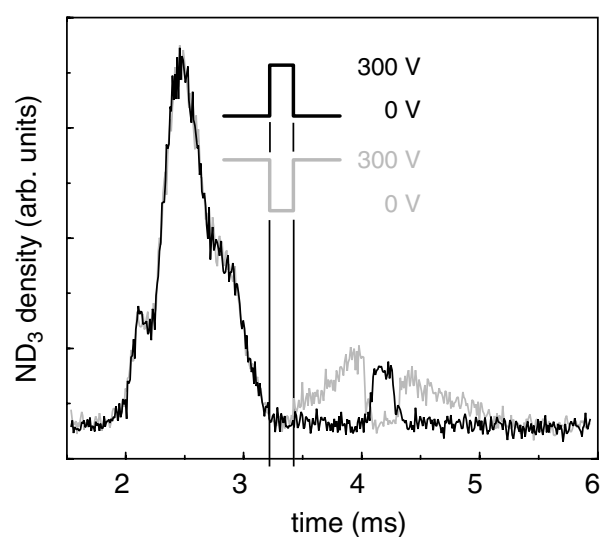

FIG. 4. Time-of-flight distribution of ammonia molecules in the detection zone with a time-varying voltage applied to the microstructured array. Two sets of independent measurements are shown in which the voltage is switched on (black curve) and off (gray curve) during a $200 \mu$ s time interval. The actual times at which the mirror is switched are indicated in the figure.

account, and interference effects can be expected in reflection from similarly corrugated mirrors.

In Fig. 4 the arrival time distributions are shown for the ammonia molecules in two independent sets of measurements in which the $300 \mathrm{~V}$ that is applied to the microstructured electrodes is being switched on (black) and off (gray) during a time interval of $200 \mu \mathrm{s}$. A block pulse showing the temporal shape of the voltage that is applied to the mirror is shown in the figure at the actual time when this voltage is applied. The actual $1 / e$ rise time of the "mirror action" in the present study is about $20 \mu \mathrm{s}$. It has been mentioned already that the observed arrival time distribution directly mimics the velocity distribution and it is thus clear that by switching the mirror on (off) for a short duration, only a small fraction of the original longitudinal velocity distribution is (is not) reflected. The arrival time distribution of the retroreflected beam recorded with the mirror switched on for $200 \mu \mathrm{s}$ corresponds to a package of molecules with a longitudinal velocity spread of about $1 \mathrm{~m} / \mathrm{s}$, or a longitudinal temperature of about $500 \mu \mathrm{K}$. It is clear that molecular beams with an extremely low longitudinal temperature, down to a few $\mu \mathrm{K}$ in the present case, can be produced via this velocity-selective reflection.

In conclusion, we have demonstrated that microstructured electrodes can be used to generate sufficiently high electric fields to manipulate polar molecules. The large electric field gradients that can be produced offer great prospects for a variety of molecular manipulation tools, including lenses, mirrors, guides, conveyer belts, decelerators, storage rings, and traps, all integrated on a compact surface area. Impinging slow beams of polar molecules on a mirror followed by rapid switching of the electric fields as demonstrated here, is a viable route to directly load molecules from a beam in microstruc- tured surface traps as well as in other microstructured devices.

This work is part of the research program of the "Stichting voor Fundamenteel Onderzoek der Materie (FOM)," which is financially supported by the "Nederlandse Organisatie voor Wetenschappelijk Onderzoek (NWO)." We acknowledge the expert technical assistance of Henrik Haak, André van Roij, and Paul Smeets, as well as useful discussions with Dr. N. J. van Druten.

[1] E. A. Hinds and I. G. Hughes, J. Phys. D 32, R119 (1999).

[2] R. Folman, P. Krüger, J. Schmiedmayer, J. Denschlag, and C. Henkel, Adv. At. Mol. Opt. Phys. 48, 263 (2002).

[3] K. S. Johnson, M. Drndic, J. H. Thywissen, G. Zabow, R. M. Westervelt, and M. Prentiss, Phys. Rev. Lett. 81, 1137 (1998).

[4] D. Müller, D. Z. Anderson, R. J. Grow, P. D. D. Schwindt, and E. A. Cornell, Phys. Rev. Lett. 83, 5194 (1999).

[5] J. Reichel, W. Hänsel, and T.W. Hänsch, Phys. Rev. Lett. 83, 3398 (1999).

[6] R. Folman, P. Krüger, D. Cassettari, B. Hessmo, Th. Maier, and J. Schmiedmayer, Phys. Rev. Lett. 84, 4749 (2000).

[7] J. Schmiedmayer, R. Folman, and T. Calarco, J. Mod. Opt. 49, 1375 (2002).

[8] J. D. Weinstein, R. deCarvalho, T. Guillet, B. Friedrich, and J. M. Doyle, Nature (London) 395, 148 (1998).

[9] H. L. Bethlem, G. Berden, F. H. M. Crompvoets, R.T. Jongma, A. J. A. van Roij, and G. Meijer, Nature (London) 406, 491 (2000).

[10] M. S. Elioff, J. J. Valentini, and D.W. Chandler, Science 302, 1940 (2003).

[11] A. J. Kerman, J. M. Sage, S. Sainis, T. Bergeman, and D. DeMille, Phys. Rev. Lett. 92, 033004 (2004).

[12] D. DeMille, Phys. Rev. Lett. 88, 067901 (2002).

[13] The planar array of equidistant electrodes is isomorphic with the geometry used in cylindrically symmetric electrostatic multipole focusers. These focusers can be viewed as working on the principle of grazing incidence reflection, and have been successfully used for state selection and beam purification in a large variety of molecular beam experiments; see Atomic and Molecular Beam Methods, edited by G. Scoles (Oxford University Press, New York, 1988), Vol. 1; Atomic and Molecular Beam Methods, edited by G. Scoles (Oxford University Press, New York, 1992), Vol. 2.

[14] J. P. Gordon, in Quantum Electronics: A Symposium, edited by C. H. Townes (Columbia University Press, New York, 1960).

[15] G. I. Opat, S. J. Wark, and A. Cimmino, Appl. Phys. B 54, 396 (1992).

[16] S. J. Wark and G. I. Opat, J. Phys. B 25, 4229 (1992).

[17] H. L. Bethlem, F. M. H. Crompvoets, R. T. Jongma, S. Y. T. van de Meerakker, and G. Meijer, Phys. Rev. A 65, 053416 (2002).

[18] P. Krüger, X. Luo, M.W. Klein, K. Brugger, A. Haase, S. Wildermuth, S. Groth, I. Bar-Joseph, R. Folman, and J. Schmiedmayer, Phys. Rev. Lett. 91, 233201 (2003). 